\title{
¿Existen respuestas jurídicas para combatir la islamofobia?
}

\section{Are there legal responses to fight Islamophobia?}

\section{Eugenia RELAÑO PASTOR}

Departamento de Derecho y Antropología del Instituto Max Planck para Antropología Social Alemania)

pastor@eth.mpg.de

Recibido 16/4/2018. Revisado y aprobado para publicación 15/6/2018

Para citar este artículo: Eugenia RELAÑO PASTOR (2018), “¿Existen respuestas jurídicas para combatir la islamofobia?" en Revista de Estudios Internacionales Mediterráneos, 24, 31-48.

Para acceder a este artículo: https://doi.org/10.15366/reim2018.24.003

\section{Resumen}

Los incidentes islamófobos han aumentado desde al año 2015 en toda Europa, según los datos de agencias europeas como la ENAR (European Network Against Racism) o la FRA (Fundamental Rights Agency), en concreto, la islamofobia ha ido dirigida especialmente hacia las mujeres musulmanas. En la primera parte del artículo se analizan las principales medidas y mecanismos en el derecho de la UE y en las instituciones internacionales para combatir este fenómeno. En la segunda parte se muestra cómo los prejuicios islamófobos están presentes en los razonamientos jurídicos de las dos altas instancias jurídicas en Europa: el Tribunal de Justicia de la Unión Europea y el Tribunal Europeo de Derechos Humanos.

Palabras claves: islamofobia, estereotipos, medidas jurídicas, Tribunales supranacionales europeos

\section{Abstract}

Since 2015, Islamophobic incidents have increased throughout Europe, according to data from European agencies such as the ENAR (European Network Against Racism) or the FRA (Fundamental Rights Agency). In particular, Islamophobia has been directed especially towards Muslim women. The first part of the article will analyze the main legal measures and mechanisms adopted by the European Union Law and the European institutions to fight this phenomenon. The second part will show how Islamophobic prejudices are present in the legal reasoning of the two Supranational European Courts: the EU Court of Justice and the European Court of Human Rights.

Keywords: Islamophobia, stereotypes, legal measures, Supranational European Courts 


\section{La islamofobia en la esfera internacional}

\section{Su reconocimiento}

La preocupación por el fenómeno de la islamofobia ha estado presente en sus diversas variantes, como el racismo antimusulmán o el odio hacia el Islam, entre las prioridades de las instituciones europeas desde principios del siglo XXI. Desde las organizaciones internacionales la islamofobia se identificó, en un primer momento, en el marco general de la lucha contra la discriminación racial ${ }^{1}$ y se incorporó progresivamente en la agenda de las organizaciones internacionales como término independiente, como puede apreciarse en las recomendaciones y resoluciones de la Asamblea Parlamentaria del Consejo de Europa ${ }^{2}$ y en los últimos documentos de la Organización para la Seguridad y Cooperación Europea (OSCE) ${ }^{3}$.

El informe más reciente de la Agencia de los Derechos Fundamentales de la Unión Europea (FRA) del año 2017, titulado Minorities and Discrimination Survey (EU-MIDIS II): Muslims-Selected findings (FRA, 2017), muestra sorprendentemente una similitud en su estructura y contenido al informe realizado por su institución predecesora, el Observatorio Europeo del Racismo y la Xenofobia (EUMC), en el año 2006. En dicho año se publica el primer trabajo exhaustivo sobre la islamofobia en el ámbito europeo: Los musulmanes en la Unión Europea: Discriminación e islamofobia 4 (EUMC, 2006). La similitud en la metodología, resultados y medidas propuestas entre los dos estudios, realizados con diez años de diferencia, nos lleva a tomar conciencia bien de las deficiencias de las medidas propuestas, de la defectuosa implementación, o de la ausencia de un compromiso serio para la erradicación de este fenómeno. La FRA alerta del peligro del estigma hacia las comunidades musulmanas y de la importancia de resaltar, en el mayor número de foros posibles, los "valores europeos" de la tolerancia, la diversidad, el respeto mutuo y la promoción de sociedades libres y pluralistas. El estigma lleva consigo la exclusión del "otro" y es terreno fértil para el florecimiento de las corrientes extremistas, tanto en la sociedad civil como en los partidos políticos $^{5}$ (Hafez, 2014; Kedikli y Akca, 2018: 14-17).

\footnotetext{
${ }^{1}$ La Comisión Europea contra el Racismo y la Intolerancia (ECRI) ha publicado varias recomendaciones dirigidas a los Estados. Algunas son específicas sobre la discriminación hacia los musulmanes, Recomendación de política general no 5 sobre la lucha contra la intolerancia y las discriminaciones hacia los musulmanes, adoptada el 16 de marzo de 2000 , y otras son recomendaciones generales en las que la islamofobia aparece como uno de los aspectos de la lucha contra el racismo, Recomendación de política general no 7 sobre la legislación nacional para luchar contra el racismo y la discriminación racial, adoptada el 13 de diciembre de 2002, o bien objeto de las medidas para erradicar el discurso del odi, Recomendación de política general no 15 sobre Líneas de Actuación para combatir el discurso de odio, adoptada el 8 de diciembre de 2015.

${ }^{2}$ Resolución de la Asamblea Parlamentaria del Consejo de Europa 1743/2010 sobre islam, islamismo e Islamofobia en Europa, Recomendación 1805 (2007) sobre la blasfemia, los insultos religiosos y el discurso de odio contra las personas por motivos religiosos, Resolución 1605 (2008) y Recomendación 1831 (2008) las comunidades musulmanas europeas confrontadas con el extremismo.

${ }^{3}$ OSCE, Decission No. 6, Tolerance and Non-discrimination, 7 December 2002, MC(10).DEC/6. Consejo de Europa y OSCE/OIDDH, UNESCO (2012), Directrices para educadores sobre la manera de combatir la intolerancia y la discriminación contra los musulmanes: Afrontar la islamofobia mediante la educacion, Varsovia, OSCE.

4 Observatorio Europeo del Racismo y la Xenofobia (EUMC) (2006), Los musulmanes en la Unión Europea: Discriminación e is/amofobia http://fra.europa.eu/en/publication/2006/highlights-eumc-report-muslims-europeanunion-discrimination-and islamophobia (acceso 21/02/2018).

5 Informe de la Agencia de los Derechos Fundamentales de la Unión Europea (FRA) (2017) Minorities and Discrimination Survey (EU-MIDIS II): $\quad$ Muslims $\quad$ - $\quad$ Selected findings, p. 7 http://fra.europa.eu/en/publication/2017/eumidis-ii-muslims-selected-findings (acceso 21/02/2018).
} 
Simultáneamente a los informes de organismos internacionales y en el ámbito europeo, en los últimos años, se han completado varios estudios relevantes sobre la discriminación que sufren las comunidades musulmanas, en concreto las mujeres musulmanas, desde organizaciones no gubernamentales. Amnistía Internacional publicaba en 2012 el informe titulado Elección y prejuicio: Discriminación de personas musulmanas en Europa en el que se conminaba a los gobiernos europeos a tomar más medidas para combatir los prejuicios y estereotipos negativos contra los musulmanes, todos ellos fomentan la discriminación, especialmente, en el colectivo femenino tanto en el ámbito educativo, como en el empleo (Amnistía Internacional, 2012: 28-83).

Conclusiones similares fueron expuestas por la Red Europea contra el Racismo (Europea Network Against Racism, ENAR) en el informe sobre el impacto de la islamofobia en las mujeres de confesión musulmana en Europa en 2016 (ENAR, 2016). La investigación que se realizó en ocho países europeos, entre los cuales no figura España, concluyó que las mujeres musulmanas experimentan las mismas desigualdades en materia de empleo y padecen las mismas violencias físicas y verbales que otras mujeres pero con el agravante de factores ligados a su religión, pertenencia étnica, nacionalidad y/o clase social (ENAR, 2016: 10-11; Bayrakli y Hafez, 2016; Taras, 2013). El informe indicaba que las mujeres musulmanas, sea en el acceso al empleo o en el mantenimiento de un puesto de trabajo, encuentran más dificultades basadas en el género, la pertenencia étnica y la religión, siendo la discriminación a la que se enfrentan a menudo vinculada a "una percepción de islamidad" y, de manera especial, a su vestimenta (ENAR, 2016: 7). Algunos estudios basados en el CV Testing evidenciaron esta discriminación interseccional (ENAR, 2016: 10-11). Por ejemplo, en Alemania, mientras el $18 \%$ de las empresas que recibieron currículos aceptaron entrevistar a mujeres con apellidos de consonancia germana, sólo un $13 \%$ lo hicieron con candidatas de origen turco y la proporción es aún más baja, un $3 \%$, en el caso de las empresas que contestaron a candidatas cuyo CV contenía una fotografía en la que llevaban velo (ENAR, 2016: 17). En Bélgica, el $44 \%$ de los empleadores piensan que el velo puede influir de manera negativa en la selección de las candidatas. $Y$, en el Reino Unido, algunas mujeres fueron cuestionadas durante las entrevistas de trabajo sobre el matrimonio y planificación familiar. Este era el caso para el $12,5 \%$ de las mujeres paquistaníes, mientras sólo el 3,3\% de las mujeres no musulmanas se enfrentaron a este tipo de preguntas (ENAR, 2016: 17-19).

También el informe de ENAR señaló ataques físicos y verbales a las mujeres musulmanas principales víctimas de crímenes de odio y agresiones verbales- las cuales están más expuestas que sus correligionarios varones, especialmente si llevan velo. También son objeto de amenazas, discurso de odio, violencia y odio en las redes sociales de manera significativa, especialmente en Facebook y Twitter (ENAR, 2016: 27-29). En Francia, por ejemplo, en el 81.5\% de los casos de violencia islamófoba registrados por el Colectivo contra la Islamofobia en 2015, las víctimas eran mujeres, principalmente, con pañuelo. Otro capítulo relevante del informe es el relativo a los prejuicios y las representaciones estereotipadas de las mujeres musulmanas difundidos tanto por los medios de comunicación como por los partidos políticos (ENAR, 2016: 26). Con frecuencia los medios presentan a las mujeres musulmanas como víctimas o como personas violentas, lo que contribuye a construir estereotipos negativos y a concebir a las mujeres musulmanas como un grupo homogéneo, negando su diversidad y heterogeneidad. Es preocupante observar la tendencia mayoritaria de los medios de comunicación a divulgar mediante ilustraciones, mensajes 
verbales o escritos, el estereotipo de la mujer con pañuelo. Esta es la imagen dominante (ENAR, 2016: 13-16) reforzada por algunos discursos políticos que reiteran la incompatibilidad entre algunas expresiones del islam y los "valores europeos". Además, se perciben a las mujeres musulmanas como un grupo homogéneo simpatizantes de la violencia doméstica, del terrorismo, de la homofobia, las desigualdades de género y de los roles tradicionales domésticos. Todo esto tiene un impacto negativo en el reconocimiento de las potenciales víctimas de discriminación y violencia, incluso por parte de los tribunales, que tienden a ser influenciados por estos estereotipos en sus resoluciones judiciales, tal y como veremos en la segunda parte de este trabajo.

\section{Propuestas jurídicas y políticas en la lucha contra la islamofobia}

En el informe del año 2006 Los musulmanes en la Unión Europea: Discriminación e islamofobia del EUMC, mencionado anteriormente, se proponían numerosas medidas en diferentes ámbitos para prevenir y erradicar la islamofobia; medidas con naturaleza jurídica como la exigencia de la transposición y aplicación efectiva de las Directivas antidiscriminación (Directiva 2000/43/CE relativa a la igualdad de trato de las personas independientemente de su origen racial o étnico y la Directiva 2000/78/CE relativa a la igualdad de trato en el empleo ${ }^{6}$ ) y medidas administrativas orientadas a obtener datos desglosados en función de los diferentes grupos de víctimas.

Otro tipo de medidas propuestas por la EUMC, con un carácter más programático que vinculante, fueron aquellas destinadas a la promoción de medidas en el ámbito laboral para la mejora de las oportunidades de empleo y la mejora en la educación a fin de evitar que alumnos pertenecientes a minorías fuesen segregados. Además, se conminaba a los Estados a revisar los libros de texto escolares para verificar que la historia de los grupos minoritarios fuse recogida de manera fidedigna y el programa de estudios incluyese de forma oficial temas como el racismo, la xenofobia, el antisemitismo y la islamofobia.

Todas estas medidas quedaron en un "wishful thinking" porque, como se señaló anteriormente, en el 2017 se tuvieron que reiterar todas las medidas pergeñadas en el informe del 2006 ante la falta de implementación. Hay que resaltar que uno de los aspectos esenciales en ambos informes es la implicación de los medios de comunicación y de los partidos políticos en el fomento o en la erradicación de la islamofobia. En el año 2003 se instó a todos los partidos políticos de Europa a aplicar la "Carta de los Partidos Políticos Europeos para una sociedad no racista", firmada el 25 de septiembre del 2003 por el presidente de la Asamblea Parlamentaria del Consejo de Europa y el presidente del Parlamento Europeo ${ }^{7}$ (ECRI, 2018). La Carta instaba a los partidos políticos para que actuasen con responsabilidad cuando se tratasen cuestiones relacionadas con la raza, el origen étnico y nacional y la religión. Lamentablemente, a la vista de los discursos de numerosos políticos europeos con motivos de la reciente llegada de refugiados, la Carta ha quedado en papel mojado ${ }^{8}$.

\footnotetext{
${ }^{6}$ Directiva 2000/78/CE del Consejo, de 27 de noviembre de 2000, relativa al establecimiento de un marco general para la igualdad de trato en el empleo y la ocupación, Diario Oficial de la Unión Europea L 303, de 2 de diciembre de 2000, pp. 16-22.

7 ECRI, Carta de los Partidos Políticos Europeos para una sociedad no racista, https://ec.europa.eu/migrantintegration/librarydoc/charter-of-political-parties-for-a-non-racist-society (acceso 19/03/2018)

${ }^{8}$ Entre otros ejemplos, el Primer Ministro Robert Fico de Eslovaquia (país en el que solo viven 10.000 musulmanes, el 0,2\% de la población total) alertó "contra la islamización de Europa" y públicamente dijo que "El islam no tiene lugar en Eslovaquia", http://www.europapress.es/internacional/noticia-primer-ministro-eslovaquia-reitera-no-quiereadmitir-musulmanes-pais-20160526070628.html (acceso 20/6/2017); en la República Checa, el "Block Against Islam"
} 
Tanto los medios de comunicación como los partidos políticos han tenido un papel muy destacado en el repunte de la islamofobia tras los ataques de Charlie Hebdo y Saint-Dennis en París en 2015 y como consecuencia de la afluencia de refugiados en Europa, en concreto, en la difusión del discurso de odio ${ }^{9}$. Por un lado, el Consejo de Europa, en el último informe del año 2016 de la ECRI, en la Recomendación General $\mathrm{n} 015$ relativa a la lucha contra el discurso de odio ${ }^{10}$, resaltó que las tendencias islamófobas se habían fusionado con los crecientes sentimientos anti-inmigrantes, como resultado de la afluencia continua de gran número de inmigrantes de países musulmanes, y alertó del potencial de minimizar todos los esfuerzos de integración realizados hasta ahora (ECRI, 2016). Esta Recomendación General también reconocía la lamentable falta de datos precisos y homogéneos sobre la extensión del uso del discurso de odio islamofóbico, incluyendo los que se divulgan en Internet y en las redes sociales y resaltaba la importancia que debía concederse a la prevención y a la lucha contra el discurso de odio ${ }^{11}$ (ECRI, 2016). También en el ámbito del Consejo de Europa, el Comité de Ministros subrayaba la necesidad urgente de prevenir el extremismo y la violencia y adoptaba para tal fin, en mayo de 2015, el Plan de Acción para la lucha contra el extremismo violento y la radicalización que conduce al terrorismo.

Es destacable, asimismo, la acción del Parlamento europeo. Tanto eurodiputados como expertos advirtieron, el 29 de junio de 2015, del peligro de la islamofobia en Europa, un problema agravado por el discurso del odio que hace mella especialmente en la comunidad musulmana. "El discurso del odio contribuye a la violencia y representa una amenaza para la sociedad democrática"12, dijo en la Eurocámara el presidente de la Comisión de Libertades Civiles de la Eurocámara. Y, por otro lado, la Comisión Europea reconoció que la lucha contra el antisemitismo y la islamofobia no es sólo una tarea de las comunidades judías y musulmanas sino de la sociedad en su conjunto. En el contexto español, en septiembre de 2015 la islamofobia se ha convertido en el principal delito de odio en España. Según los informes policiales y del Ministerio del Interior, que hicieron públicos a través del periódico "El País", los ataques contra el islam y contra los musulmanes representan el $40 \%$ de los delitos de odio registrados por la policía ${ }^{13}$ (Ministerio de Interior, 2016: 19).

logro reunir 145.000 firmas en una petición contra la entrada de inmigrantes musulmanes, https://bajurtov.wordpress.com/2015/01/08/excelente-otro-politico-anti-islam-en-la-republica-checa-insta-a-lagente-a-pasear-a-los-perros-y-los-cerdos-cerca-de-las-mezquitas/ (acceso 20/6/2017) o el Primer Ministro húngaro, Viktor Orbán, manifestó con motivo de la llegada de refugiados a la frontera húngara : "No vemos a los musulmanes como refugiados sino como invasores", https://www.elconfidencial.com/mundo/2018-01-08/hungria-orbanrefugiados-islam-invasores 1502709 (acceso 20/6/2017)

${ }^{9}$ Vid. Informe de la Agencia de los Derechos Fundamentales de la Unión Europea (FRA), Minorities and Discrimination Survey (EU-MIDIS II): Muslims - Selected findings (2017). (acceso 21/02/2018)

${ }^{10}$ Comisión Europea contra el Racismo y la Intolerancia (ECRI), Recomendación General no 15 relativa a la lucha contra el discurso de odio, adoptada Estrasburgo, adoptada el 8 de diciembre de 2015, Estrasburgo, 21 de marzo de 2016.

${ }^{11}$ En este mismo sentido, vid. Informe de la Agencia de los Derechos Fundamentales de la Unión Europea (FRA) Minorities and Discrimination Survey (EU-MIDIS II), 2017, op. cit.

12 http://www.dw.com/es/advierten-aumento-de-la-islamofobia-en-europa/a-18550531 (acceso 16/03/2018)

${ }^{13}$ http://politica.elpais.com/politica/2015/09/06/actualidad/1441569646 31 615.html No obstante, en el año 2016, según el Ministerio de Interior, los incidentes relacionados con las "creencias o prácticas religiosas", a diferencia del año 2015, han sufrido un descenso del 32,9\%. Es de señalar que el concepto "islamofobia" no aparecer como tal, aunque sí aparece la categoría de "antisemitismo". 
Todas estas llamadas de atención han sido medidas programáticas, recomendaciones a los Estados que no han llevado consigo una correlativa responsabilidad en su cumplimiento. Las principales medidas jurídicas de lucha contra la islamofobia y el odio hacia los musulmanes han estado dirigidas tanto a los Estados como a los medios de comunicación, partidos políticos y sociedad en su conjunto. Desde el punto de vista jurídico, se ha instado a los Estados a ratificar el Protocolo Adicional al Convenio sobre la Ciberdelincuencia relativo a la penalización de actos de índole racista y xenófoba cometidos por sistemas informáticos, de 28 de enero de 2003 (ratificado por España 2015) y a retirar cualquier reserva formulada al artículo 4 de la Convención sobre la Eliminación de todas las Formas de Discriminación Racial y al artículo 20 del Pacto Internacional sobre Derechos Civiles y Políticos, así como a reconocer la competencia del Comité para la Eliminación de la Discriminación Racial para recibir y examinar las comunicaciones enviadas por grupos o particulares conforme al artículo 14 de la mencionada Convención.

En el ámbito específicamente europeo se ha recomendado a los Estados impulsar la Propuesta de Directiva del Consejo por la que se aplica el principio de igualdad de trato entre las personas independientemente de su religión o convicciones, discapacidad, edad u orientación sexual del $2008^{14}$. Por otro lado, la Comisión Europea debería iniciar procedimientos de infracciones sobre el incumplimiento de la Directiva sobre el empleo (2000/78/CE) cuando exista una discriminación sistemática por motivos de religión y creencias, como son las restricciones al uso de símbolos religiosos por motivos de estricta neutralidad en el trabajo. Además, la Comisión Europea debería aplicar procedimientos de infracción contra los Estados miembros que no han transpuesto aun la Decisión marco 2008/913/JAl del Consejo, de 28 de noviembre de 2008, relativa a la lucha contra determinadas formas y manifestaciones de racismo y xenofobia mediante el Derecho penal ${ }^{15}$.

Existen otro grupo de medidas adoptadas por la ECRI, sin vinculación directamente jurídica para los Estados, pero de gran utilidad práctica como la propuesta a la FRA y al Instituto para la Igualdad de Género para que cooperen y presenten informes sobre la discriminación múltiple que afecta a las mujeres con estadísticas e información desagregada sobre género, origen étnico y religión (ECRI, 2016: 22). La ECRI también ha resaltado la necesidad de estudiar las condiciones que fomentan el empleo del discurso de odio y evaluar su difusión y el daño que produce, a fin de desincentivar e impedir su empleo. Para ello se deberían articular herramientas fiables para la recogida de datos sobre discurso de odio que puedan ser utilizadas por organismos públicos y que se no limite, exclusivamente, al ámbito de la justicia penal (ECRI, 2016: 22). Con estos datos, recabados y desglosados, las autoridades públicas deberían elaborar estrategias que permitiesen abordar este problema. Otra faceta importante para erradicar los discursos de odio contra la población musulmana es concienciar a los ciudadanos sobre la importancia de respetar el pluralismo y los peligros que supone el discurso de odio. No solo para demostrar que son inaceptables, y que las premisas en que las se basan son falsas sino, también, para impedir el empleo de este lenguaje. De este modo se combate la información maliciosa, la difusión de estereotipos negativos y la estigmatización. También se debe exhortar a las figuras públicas y, en especial, a los líderes políticos, religiosos de las comunidades a que reaccionen con presteza ante el discurso de odio, para condenarlo y para realzar los valores contra los que se atentan. Todos los

\footnotetext{
14 Propuesta de Directiva del Consejo por la que se aplica el principio de igualdad de trato entre las personas independientemente de su religión o convicciones, discapacidad, edad u orientación sexual \{SEC(2008) 2180\} \{SEC(2008) 2181\}, http://eur-lex.europa.eu/legal-content/ES/ALL/?uri=CELEX\%3A52008PC0426 (acceso 16/03/2018)

15 Decisión Marco 2008/913/JAl del Consejo, de 28 de noviembre de 2008, relativa a la lucha contra determinadas formas y manifestaciones de racismo y xenofobia mediante el Derecho penal, publicado DOUE, núm. 328, de 6 de diciembre de 2008, pp. 55-58.
} 
partidos políticos deberían firmar la Carta de los Partidos Políticos Europeos para una sociedad no racista y adoptar los pertinentes códigos de conducta que prevean la suspensión y otras sanciones en caso de infracción de sus disposiciones (ECRI, 2016: 55). No solo los políticos, también los medios de comunicación deben de dotarse de mecanismos de autorregulación y adoptar los pertinentes códigos de conducta en materia de discurso de odio. Se deben promover el seguimiento y la condena del empleo y difusión del discurso de odio; fomentar la adopción, si procede, de restricciones de contenido, instrumentos de filtrado de términos y otras técnicas similares, así como animar a que se organice la formación adecuada de directores, periodistas y demás personal de los medios de comunicación en cuanto a la naturaleza del discurso de odio y al modo de hacerle frente (ECRI, 2016: 59-64).

En cuanto a los poderes públicos, estos deben retirar todo apoyo económico o de otra índole a los partidos políticos y otras organizaciones que empleen discurso de odio y determinar las responsabilidades concretas de los autores de discurso de odio, prestadores de servicios de internet, foros y hosts de internet, intermediarios on line, plataformas de redes sociales, moderadores de blogs etc. Las administraciones deben velar por que se otorguen facultades, sujetas a autorización judicial, para requerir la supresión del discurso de odio de material accesible en internet y para bloquear sitios en los que se empleen. Todo ello debe acompañarse con una formación adecuada que facilite el intercambio de buenas prácticas entre jueces, abogados y funcionarios que se ocupen de casos relativos al discurso y crímenes de odio ECRI, 2016: 11- y 68). No hay que olvidar que la finalidad de políticas de lucha contra el discurso del odio debe estar encaminada a la protección de las víctimas de estos discursos. Se tienen que establecer protocolos específicos de ayuda, asesoramiento y orientación legal, a fin de superar cualquier trauma o sentimiento de vergüenza que hayan sufrido y velar por una efectiva reparación de los daños causados bien por los procedimientos administrativos, civiles o penales. El objetivo es evitar que el miedo, la ignorancia, los obstáculos físicos-emocionales o la falta de medios, les impidan ejercer sus derechos (ECRI, 2016: 8-13).

En definitiva, las instancias internacionales, y más concretamente las europeas, llevan realizando durante la última década una labor de reconocimiento de la importancia de tomar en serio la islamofobia, lo cual viene a constatar el "reproche" fruto de la desaprobación social hacia este tipo de fenómeno. No obstante, la mayor parte de las medidas sugeridas y exigidas a los Estados se han articulado mediante políticas públicas e iniciativas de las distintas administraciones, sin que se haya consolidado un derecho vinculante específico para combatir la islamofobia en todas sus dimensiones.

\section{Islamofobia en los Tribunales supranacionales europeos}

\section{Tribunal de Justicia de la Unión Europea}

El pasado 14 de marzo de 2017 el Tribunal de Justicia de la Unión Europea (TJUE) decidió los dos primeros casos sobre discriminación religiosa en el empleo, ambos relativos a la posible discriminación de las prohibiciones del uso visible de cualquier signo político, filosófico o religioso en el trabajo 
(Palomino, 2016 y Relaño, 2018): los casos Achbita y Bougnaoui ${ }^{16}$. El TJUE sostuvo que una norma interna de una empresa que prohíbe el uso visible de cualquier signo político, filosófico o religioso no constituye una discriminación directa. No obstante, en ausencia de tal norma, la voluntad de un empresario de tener en cuenta los deseos de un cliente de que los servicios de dicho empresario no sigan siendo prestados por una trabajadora que lleva un pañuelo islámico, no puede considerarse un requisito profesional que permita descartar la existencia de una discriminación ${ }^{17}$.

En el primer supuesto, Achbita (c. G4S Secure Solutions), el TJUE resolvió a favor de la empresa, entendiendo que una norma interna de una empresa que prohíbe el uso visible de cualquier signo político, filosófico o religioso no constituye una discriminación directa y que, en el caso de que pueda constituir una discriminación indirecta, está justificada de forma objetiva. En este caso, la trabajadora Samira Achbita, de confesión musulmana, empleada desde el año 2003, comunicó a su empleador en 2006 después de una baja laboral por enfermedad, que pretendía reanudar la actividad laboral llevando velo islámico, el día 15 de mayo de 2006. El día 29 de ese mismo mes, el comité de empresa aprobó una modificación de un reglamento interno, que entró en vigor el 13 de junio de 2006, en el que prohibía a los trabajadores llevar símbolos de carácter político, filosófico o religioso en el lugar de trabajo. Samira Achbita fue despedida el 12 de junio de 2006 por su insistente voluntad de llevar velo islámico. La Sra. Achbita impugnó el despido en los tribunales belgas. El TJUE consideró que la norma interna de la empresa tiene por objeto prohibir el uso de todo tipo de signos que hagan visibles las creencias políticas o religiosas de los trabajadores indistintamente del tipo de ideología o religión. Esta norma se impone por tanto de forma neutral y generalizada, y es por este motivo que no se puede decir que se trate de una norma de discriminación directa que establezca una diferencia de trato basada directamente en la religión islámica.

En el segundo caso (Bougnaoui y $A D D H$ ), el TJUE resolvió que, en el caso de que el despido de la Sra. Bougnaoui no se hubiera basado en una norma interna de la empresa se podría valorar el requisito del art. 4.1 de la Directiva 78/2000/CE, siempre y cuando el objetivo sea legítimo y el requisito proporcionado ${ }^{18}$. El despido de la trabajadora de la empresa donde trabajaba (Micropole) se produjo tras la queja de un cliente. La trabajadora había llevado el velo islámico durante todo el periodo de prácticas en la empresa efectuadas durante el año 2008. Al finalizar las prácticas la trabajadora fue contratada por la empresa a tiempo indefinido y usó el velo islámico en su lugar de trabajo, sin tener ningún tipo de problema antes de que se produjera la queja. Tras esta queja, la empresa le reiteró el principio de neutralidad a la trabajadora pidiéndole que dejara de llevar el velo. La trabajadora se negó y fue despedida. Bougnaoui impugnó el despido ante los tribunales franceses.

No es el lugar para analizar en profundidad las cuestiones jurídicas de fondo que plantean ambas sentencias. Sin embargo, destacaremos ciertos prejuicios islamófobos que se traslucen en los

\footnotetext{
${ }^{16}$ Sentencias en los asuntos C-157/15 Achbita, Centrum voor gelijkheid van kansen en voor racismebestrijding / G4S Secure Solutions y C-188/15 Bougnaoui y Association de défense des droits de l'homme (ADDH) / Micropole Univers.

17 El Tribunal de Justicia tenía que considerar los conceptos de discriminación directa e indirecta recogidos en la Directiva 2000/78/CE del Consejo, de 27 de noviembre de 2000, relativa al establecimiento de un marco general para la igualdad de trato en el empleo y la ocupación (Diario Oficial de la Unión Europea L 303, de 2 de diciembre de 2000, 16-22).

18 Artículo 4.1 de la Directiva 78/2000/CE sobre los requisitos profesionales: 1 . No obstante lo dispuesto en los apartados 1 y 2 del artículo 2, los Estados miembros podrán disponer que una diferencia de trato basada en una característica relacionada con cualquiera de los motivos mencionados en el artículo 1 no tendrá carácter discriminatorio cuando, debido a la naturaleza de la actividad profesional concreta de que se trate o al contexto en que se lleve a cabo, dicha característica constituya un requisito profesional esencial y determinante, siempre y cuando el objetivo sea legítimo y el requisito, proporcionado.
} 
razonamientos jurídicos, en concreto, en el caso Achbita. En ambas sentencias el Tribunal de Justicia de la Unión Europea valida las conclusiones de sus correspondientes Abogados Generales (AG), Kokott y Sharpston en su totalidad ${ }^{19}$.

Para nuestro análisis es destacable la Opinión de la Abogada General (AG) Kokott en el caso Achbita. En los primeros párrafos señala que "los problemas jurídicos en torno al velo islámico son un ejemplo de la cuestión fundamental sobre el grado de diferencia y diversidad que debe admitir en su seno una sociedad europea abierta y plural y, a la inversa, qué grado de adaptación puede exigirse a determinadas minorías" ${ }^{20}$. Es decir, la premisa de partida para la AG Kokott es cuánta diversidad puede permitirse y admitirse en Europa, dando por supuesto que la diferencia viene de fuera y la sociedad europea, teóricamente homogénea, tuviera que tolerar al "otro", al diferente. Este punto de partida va a influir en toda la argumentación jurídica posterior, por ejemplo, en el concepto de religión. En palabras de AG Kokott, “(...) a diferencia del sexo, el color de la piel, el origen étnico, la orientación sexual, la edad y la discapacidad de una persona, la práctica religiosa no es tanto una circunstancia invariable como un aspecto de la vida privada, en el cual además pueden influir voluntariamente los trabajadores afectados. Mientras que un trabajador no puede «dejar en el guardarropa» su sexo, su color de piel, su origen étnico, su orientación sexual, su edad ni su discapacidad al acceder a las instalaciones de su empresario, sí se le puede exigir un cierto recato en el trabajo con respecto al ejercicio de su religión, ya sea en relación con sus prácticas religiosas, sus comportamientos motivados por la religión o (como aquí sucede) su forma de vestir"21. De acuerdo con el razonamiento de la AG Kokott, dado que uno puede elegir una religión, cualquier limitación de la manifestación de la libertad religiosa seria adecuada porque la religión no es una característica inmutable. Como señala Vickers, este argumento es altamente cuestionado (Vickers, 2016). Incluso cuando la religión sea una opción voluntaria, el coste que soporta cualquier creyente al renunciar a un aspecto clave de su identidad es importante. Existen otro tipo de características personales relativas a la identidad que pueden elegirse, como el embarazo y la identidad como madre, y no por ello podría justificarse una discriminación basada en el género. En contraste con la posición de AG Kokott, la Abogada General Sharpston sostuvo en su Opinión del caso Bougnaoui exactamente lo contrario: "sería totalmente incorrecto suponer que, mientras que el sexo y el color de la piel siempre acompañan a las personas, de alguna manera no sucede así con su religión"22.

\footnotetext{
${ }^{19}$ El Tribunal de la Unión Europea publica las conclusiones de los Abogados Generales, los cuales emiten un dictamen jurídico sobre la cuestión prejudicial que se somete a consideración del Tribunal. La cuestión prejudicial no es propiamente un recurso ante el Tribunal sino un procedimiento de cooperación entre el Tribunal de Justicia y los Tribunales nacionales. Los tribunales nacionales se dirigen para resolver dudas sobre una norma comunitaria que debe interpretarse pero cuya validez no se discute (artículo 267 del Tratado de Funcionamiento de la Unión Europea). En general, el Tribunal de la Unión Europea suele validar las conclusiones de los Abogados Generales.

${ }^{20}$ Asunto C-157/15 Conclusiones, par. 3.

${ }^{21}$ Asunto C-157/15 Conclusiones, par. 116.

22 “(...) para un miembro practicante de una religión, la identidad religiosa forma parte integrante de su ser. Los requisitos impuestos por la fe — su disciplina y las normas sobre la manera en que los adeptos deben llevar su vidano son elementos que deban aplicarse cuando uno no esté trabajando (digamos, por la noche y durante los fines de semana para quienes tengan un trabajo de oficina) y que puedan dejarse discretamente de lado en horas de trabajo. Según las reglas particulares de la religión en cuestión y el grado en el que un determinado individuo sea practicante, alguno de dichos elementos puede, por supuesto, no ser de obligado cumplimiento para dicho individuo y, por lo tanto, ser negociable. Pero sería totalmente incorrecto suponer que, mientras que el sexo y el color de la piel siempre
} 
A mi juicio, el derecho de la Unión Europea debería proteger las manifestaciones de las creencias fundamentales no porque sean inmutables, sino por su importancia y centralidad en la dignidad del individuo. No debe olvidarse que la UE se basa, entre otros principios, en el valor del respeto de la dignidad y la libertad del ser humano (artículo 2 del Tratado de la Unión Europea, TUE). El hecho de que la intensidad de la observancia religiosa pueda fluctuar a lo largo de la vida de una persona no significa que la fe o las creencias no merezcan su debida protección. Lamentablemente, AG Kokott sugiere que existen ciertos "grados" en el tema religioso al señalar que una religión "moderada" es más apropiada para el ámbito laboral. Este tipo de condescendencia sobre lo que significan las "creencias religiosas" para la vida de una persona es otra manifestación más del tipo de paternalismo invasivo hacia las mujeres musulmanas en el que ha incurrido igualmente el Tribunal de Estrasburgo. Del razonamiento jurídico de Achbita se deduce que la conciencia de todo creyente, muy en concreto de las mujeres musulmanas, se enfrenta con el dilema de dejar su religión a las puertas del trabajo o quedarse excluidas del mundo laboral.

Es también posible detectar un rechazo a ciertas manifestaciones de la libertad religiosa que trasluce una islamofobia soterrada. $Y$ se hace mediante la defensa de una estricta idea de neutralidad que afecta particularmente más a las mujeres musulmanas que a cualquier otro grupo religioso. AG Kokott defiende una neutralidad secularista que implica una restricción al uso de símbolos religiosos personales. En su Opinión indica que "si bien la actividad de una recepcionista puede desempeñarse igual de bien con velo que sin él, también es cierto que puede formar parte del contexto en que se lleva a cabo observar las normas de indumentaria impuestas por el empresario (por ejemplo, la obligación de llevar una ropa de trabajo o un uniforme, o bien la eventual prohibición de llevar símbolos religiosos, políticos o filosóficos visibles), de manera que la trabajadora desarrolla su trabajo en un contexto en que debe renunciar a su velo (...) una empresa (como G4S) puede optar por una política de estricta neutralidad religiosa y de convicciones y, con vistas a la realización de esa imagen, exigir a sus trabajadores como requisito profesional la correspondiente presencia neutral en el puesto de trabajo" 23 . Es decir, en el juicio de proporcionalidad que implica cualquier ponderación de intereses y derechos en conflicto, el elemento de la neutralidad religiosa prevalece sobre los intereses legítimos de las trabajadoras afectadas porque no ha excedido los límites del margen de apreciación empresarial (es decir, el código de vestir que ha impuesto el empresario). Como afirma Brems, extender la neutralidad al sector privado significa "un gran salto" jurídico porque "la neutralidad puede encubrir fácilmente prejuicios" (Brems, 2017). Si la política de neutralidad se rige por las demandas y expectativas de los clientes, -como indica AG Sharpston en Bougnaoui-, "cuando la propia actitud del cliente puede indicar que éste tiene un prejuicio basado en uno de los «criterios prohibidos», como la religión, me parece particularmente peligroso que se dispense al empresario del deber de cumplir con la obligación de la igualdad de trato para complacer tal prejuicio" 24 .

El impacto de estos dos casos en la jurisprudencia de los tribunales europeos nacionales está aún por evaluar. Sin embargo, es necesario recordar el papel del Tribunal de Justicia de la Unión Europea como intérprete supremo del derecho de la Unión Europea. Como órgano encargado de proporcionar normas comunes y orientación para aplicar la legislación de la UE en una Europa pluralmente religiosa y diversa, el Tribunal ha enviado un mensaje de exclusión y de intolerancia. El resultado en estos dos

acompañan a las personas, de alguna manera no sucede así con su religión (...), Asunto C-188/15, Conclusiones, par. 118.

${ }^{23}$ Asunto C-188/1, Conclusiones, par. 76.

${ }^{24}$ Asunto C-188/1, Conclusiones, par. 133. 
casos, y en los que sigan sus razonamientos jurídicos, es la exclusión de un tipo concreto de diversidad religiosa, la de las mujeres musulmanas en el ámbito laboral, las cuales quedan apartadas del sector privado empresarial al arbitrio de la política de neutralidad que desee imponer el empresario. Los efectos para la cohesión social y la repercusión estructural en el mercado laboral pueden ser devastadores para la efectiva integración de las mujeres musulmanas.

\section{Tribunal Europeo de Derechos Humanos}

El Tribunal Europeo de Derechos Humanos (TEDH), también conocido como Tribunal de Estrasburgo, es el organismo judicial de control de la vigencia de los derechos reconocidos en el Convenio Europeo de Derechos Humanos y se ha convertido en el paradigma para las decisiones judiciales nacionales en los Estados miembros del Consejo de Europa. La actividad del Tribunal Europeo respecto del derecho de libertad religiosa, recogido en el artículo 9 del Convenio ${ }^{25}$, cuya redacción está inspirada en el artículo 18 de la Declaración Universal de los Derechos Humanos, ha sido muy amplia y no ha estado exenta de polémica (Murdoch, 2012).

Una tendencia jurisprudencial del TEDH es una concepción de la libertad religiosa desde un liberalismo que prima la autonomía del individuo frente a todo tipo de coacción (libertad religiosa negativa). Como herencia del enfoque del liberalismo clásico de los derechos humanos, se ha reconocido la primacía de la conciencia individual sobre la coacción estatal y para ello el Tribunal ha diferenciado el forum internum del forum externum (Petkoff, 2012). El foro externo, es decir la manifestación de las conductas exteriores motivadas por las convicciones religiosas, no se protege de igual manera para todos los creyentes. Para aquellos pertenecientes a la confesión musulmana, el Tribunal ha adoptado un sesgo paternalista, como veremos a continuación. Otra tendencia del Tribunal de Estrasburgo ha sido la prevalencia de la neutralidad estatal sobre el ejercicio de la autonomía personal. Países como Francia y Turquía, caracterizados por una fuerte neutralidad estatal convertida en principios fundamentales del Estado, han protagonizado numerosas sentencias en las que se restringen la manifestación de la libertad religiosa de las minorías religiosas, en especial, de las musulmanas.

En todo caso, el carácter neutral del Estado debería implicar el respeto por las comunidades que coexisten en el territorio. El Estado aparece como el "arbitro" entre los "competidores" religiosos y el garante de un pluralismo de hecho. Este fue el enfoque adoptado el Tribunal de Estrasburgo en el caso Kokkinakis contra Grecia ${ }^{26}$ cuando afirmaba que el pluralismo es, en su dimensión religiosa, uno de los elementos más importantes que conforman la identidad de los creyentes y de su concepción de la vida, además, de un activo valioso para ateos, agnósticos, escépticos ${ }^{27}$. En este sentido, el pluralismo

\footnotetext{
${ }^{25}$ Artículo 9 CEDH: "1. Toda persona tiene derecho a la libertad de pensamiento, de conciencia y de religión; este derecho implica la libertad de cambiar de religión o de convicciones, así como la libertad de manifestar su religión o sus convicciones individual o colectivamente, en público o en privado, por medio del culto, la enseñanza, las prácticas y la observancia de los ritos. 2. La libertad de manifestar su religión o sus convicciones no puede ser objeto de más restricciones que las que, previstas por la ley, constituyan medidas necesarias, en una sociedad democrática, para la seguridad pública, la protección del orden, de la salud o de la moral públicas, o la protección de los derechos o las libertades de los demás".

${ }^{26}$ STEDH, Kokkinakis c. Grecia, Sentencia de 25 de mayo de 1993, Application no. 14307/88.

27 "Tal y como la protege el artículo 9, la libertad de pensamiento, de conciencia y de religión constituye una de las bases de una "sociedad democrática" en el sentido del Convenio. Figura en su dimensión religiosa entre los elementos
} 
es el medio y el fin de una verdadera libertad religiosa, así como un bien básico para una democracia saludable. Si bien el respeto por el pluralismo fue fundamental en Kokkinakis o en Iglesia Metropolitana de Besarabia y otros contra Moldavia ${ }^{28}$, en los llamados casos islámicos, el pluralismo deja de ser consubstancial a nuestra sociedad y queda limitado con el argumento de la protección del orden y de la seguridad pública (Evans, 2000 y Martínez-Torrón, 2003) o por nuevos límites de creación jurisprudencial como el concepto de "vivir juntos" creado por el TEDH en el caso de S.A.S contra Francia ${ }^{29}$.

\section{Autonomía y dignidad de los creyentes musulmanes}

Existen varias manifestaciones de prejuicios islamófobos en los razonamientos jurídicos de las sentencias del Tribunal de Estrasburgo. La primera manifestación sería la negación de la autonomía personal de los creyentes musulmanes, en concreto de las mujeres que llevan el velo. Entre las decisiones del TEDH relativas al uso del pañuelo o velo islámico, destacan Dahlab c. Suiza ${ }^{30}$, Leyla Sahin c. Turquía ${ }^{31}$, Kervanci c. Francia ${ }^{32}$ y Dogru c. Francia ${ }^{33}$, todas ellas relativas a la prohibición del uso del velo islámico no integral en el contexto escolar. A estas hay que añadir las tres sentencias sobre la prohibición del velo integral en S.A.S c. Francia, Belcacemi y Ousar c. Bélgica y Dakir c. Bélgica. En este tipo de casos el uso de un determinado símbolo religioso personal -el hiyab o el velo integral- va en menoscabo de los principios democráticos de las sociedades europeas, es decir, son considerados en sí mismos "antidemocráticos".

En el caso Dahlab, relativo a la prohibición de llevar velo por parte de una profesora de escuela primaria conversa al islam, el TEDH sostuvo que no prevalece el derecho de libertad de creencias sobre el interés del Estado en la protección de los derechos y libertades de los demás y la conservación del orden y la seguridad públicas: "Por lo tanto, parece difícil conciliar el uso de un islámico pañuelo con el mensaje de tolerancia y respeto por los demás" ${ }^{34}$. El Tribunal se arroga la competencia de valorar el significado de un símbolo religioso, lo cataloga como antidemocrático y lo contrapone al principio constitucional de neutralidad. De este modo, la titular del derecho de libertad religiosa, la mujer musulmana que desea expresar su libertad religiosa mediante el uso de un símbolo religioso personal, queda silenciada ante el peso de los principios del secularismo y los valores democráticos. Igualmente en Sahin, el Tribunal señaló que "(...) es el principio de secularismo, tal como lo ha entendido el Tribunal Constitucional, lo que subyace a la prohibición del uso de símbolos religiosos en las universidades" 35 .

En Dogru, un caso en el que una estudiante musulmana se negó a quitarse el pañuelo en las clases de educación física y deportes fue expulsada del colegio, el TEDH determinó que “(...) Cuando se trata de cuestiones relativas a la relación entre el Estado y las religiones, sobre las cuales la opinión en una

más esenciales de la identidad de los creyentes y de su concepción de la vida, pero es también un bien precioso para los ateos, los agnósticos, los escépticos o los indiferentes. Es una manifestación del pluralismo, claramente conquistado en el curso de siglos, consubstancial a nuestra sociedad", par. 31.

${ }^{28}$ STEDH, Iglesia Metropolitana de Besarabia y otros c. Moldavia, Sentencia de 3 diciembre de 2001, Application 31/12/2001, par. 116.

${ }^{29}$ STEDH, S.A.S. c. Francia, Sentencia de 1 de julio de 2014, Application no. 43835/11.

${ }^{30}$ STEDH, Dahlab c. Suiza, Sentencia de 15 de febrero de 2001, Application no. 42393/98.

${ }^{31}$ STEDH, Leyla Sahin c. Turquía, Sentencia de 10 de noviembre de 2005, Application no. 44774/98.

${ }^{32}$ STEDH, Kervanci c. Francia, Sentencia de 4 de diciembre de 2008, Application no. 31645/04.

${ }^{33}$ STEDH, Dogru c. Francia, Sentencia de 4 de diciembre de 2008, Application no. 27058/05.

${ }^{34}$ STEDH, Dahlab c. Suiza, par. 1.

${ }^{35}$ STEDH, Sahin c. Turquia par. 116. 
sociedad democrática puede diferir razonablemente, se debe otorgar especial importancia al papel del órgano nacional en la toma de decisiones. Este será el que decida sobre la regulación de uso de símbolos religiosos en las instituciones educativas (...."136. Asimismo, también, merece la pena destacar que en Dogru el Tribunal de Estrasburgo sugiere que se ha tomado en cuenta "los derechos y libertades de los demás" cuando, en realidad, se ignoró la autonomía y la voz de la alumna Begin Dogru al rechazarse su sugerencia de usar una bandana, en lugar del velo a fin de cumplir con las reglas internas de la escuela.

En todos estos casos el Tribunal se preocupa por la preservación de los principios constitucionales del Estado -la democracia, la tolerancia, la laicidad, el orden público y los derechos y la libertad de los demás- ignorando los argumentos de las solicitantes respecto a sus nomos religiosos y a su autonomía personal. Cuando se trata de mujeres musulmanas la negación de la autonomía personal va, además, acompañada de un paternalismo abusivo que trata de proteger a la mujer de su propia autoafirmación, libre e individual, sobre lo que es digno para sí misma (Howard, 2012: 160). La jueza Tulkens, en su opinión disidente en Sahin, al preguntarse si el hiyab se ajusta a los valores democráticos de la igualdad de género y de la tolerancia enfatizaba: "(...) Por lo tanto, se considera que la prohibición de usar el velo es promover la igualdad entre hombres y mujeres. Sin embargo, ¿cuál es, de hecho, la conexión entre la prohibición y la igualdad sexual? La sentencia no lo dice. De hecho, ¿cuál es el significado de usar el pañuelo en la cabeza? ... Lo que falta en este debate es la opinión de las mujeres, tanto de quienes usan el pañuelo como de quienes eligen no hacerlo"37.

La negación de las voces de las mujeres musulmanas no solo implica el paternalismo de convertirse en portavoz de aquellas que "no tienen voz" sino que, además, significa la cosificación de la mujer, al desposeerla de su propia singularidad. El Estado se convierte de esta manera en el portavoz de cómo perciben y viven el islam estas mujeres. Es la negación de lo "otro" y la suplantación de lo que quiere la sociedad que sea eso "otro".

No obstante, la lógica del Tribunal de Estrasburgo en los casos mencionados ha sido corregida en sentencias posteriores hasta llegar a reconocer que la autonomía de las mujeres ha de ser tomada en consideración (Brems, 2013: 2-14; Brems, 2014; O’Neill et al, 2014; Vakulenko, 2012). Así se señaló tanto en Eweida y otros contra Reino Unido y como en S.A.S ${ }^{38}$. Paradójicamente, en Eweida que implicaba la exhibición de un símbolo cristiano, el Tribunal dio peso en la argumentación jurídica a la autonomía de la creyente cristiana, en concreto, a su visión personal de su propia libertad religiosa. Por el contrario, en S.A.S, si bien se le dio igualmente voz a la mujer musulmana sobre lo que significaba para ella la exteriorización de su libertad religiosa, no fue determinante para la decisión de la sentencia ${ }^{39}$. En todo caso, lo destacable es que el Tribunal de Estrasburgo afirmo en Eweida que el

\footnotetext{
${ }^{36}$ STEDH, Dogru c. Francia, par. 63.

${ }^{37}$ STEDH, Sahin c. Turquia, Opinión discrepante de la jueza Tulkens, par 11.

${ }^{38}$ En Eweida y otros c. Reino Unido, Application num. 48420/10, 36516/10, 51671/10, de 15 de enero de 2013, relativo al uso de llevar una cruz visiblemente en el trabajo, el Tribunal consideró que el comportamiento de la Sra. Eweida formaba parte del contenido del artículo 9, por tanto, era parte de su manifestación religiosa, en forma de culto, práctica y observancia.

${ }^{39}$ En S.A.S, la demandante, una mujer de nacionalidad francesa, declaró utilizar -aunque con carácter esporádico- el velo integral como parte de la manifestación externa de su cultura y su religión musulmana.
}

REIM № 24 (junio 2018)

ISSN: 1887-4460 
deseo de manifestar las propias creencias es un derecho fundamental porque una sociedad democrática saludable necesita tolerar y sostener el pluralismo y la diversidad, pero también por el valor que tiene la religión para la persona que la ha convertido en el eje central de su vida, en consecuencia, tiene el derecho de comunicar esta creencia a los demás. Como indicó el antiguo Comisario de Derechos Humanos, Thomas Hammarberg, Human Rights League y Human Rights Watch, "la prohibición del velo, en lugar de ayudar a las mujeres que son obligadas a llevarlo, limitará su capacidad para buscar asesoramiento y ayuda. El primer efecto de esta medida va a ser confinar a las mujeres con velo en sus hogares en vez de liberarlas" (Human Rights Watch, 2010 y Human Rights League, 2010 $)^{40}$. Este tipo de iniciativas legislativas son contraproducentes para la igualdad de género (Relaño, 2015 y Howard, 2012).

\section{Prejuicios negativos}

La segunda manifestación de islamofobia es la tendencia a interpretar el islam y a los musulmanes como una realidad monolítica y compacta (Marranci, 2005; Halliday, 1999). Las sociedades europeas siguen atribuyendo a los musulmanes unas singularidades predefinidas. Esta visión del islam no lleva consigo necesariamente prejuicios islamófobos. Sin embargo, el imaginario social europeo sigue atribuyendo a los musulmanes una singularidad predefinida y estereotipos negativos (Bravo, 2011). Bajo la categoría de musulmán se incluye a personas esencialmente religiosas, en ocasiones inintegrables, debido al fuerte arraigo cultural en sus propias comunidades (Rana, 2007). Se impone una determinada caracterización negativa a los musulmanes, al tiempo que se ignora la autodefinición que hacen los musulmanes de sí mismos (Bectovic, 2011).

En los casos del uso del pañuelo o velo islámico, el Tribunal ha construido una imagen negativa del pañuelo en la cabeza, calificándolo como símbolo que no cumple con los valores de la democracia europea (Peroni, 2014: 201-2013). Por ejemplo, en Dahlab, la Gran Sala se refirió al uso del pañuelo en la cabeza como un "poderoso símbolo externo" que "parecía imponerse a las mujeres por un precepto religioso difícil de conciliar con el principio de igualdad de género". Afortunadamente en S.A.S, diez años después de Sahin, el Tribunal ya es consciente de los estereotipos negativos que existen en la sociedad sobre las mujeres musulmanas como mujeres oprimidas que necesitan protección y corrige los argumentos del gobierno francés relativos a la igualdad de género. El Tribunal de Estrasburgo, por el contrario, sostiene que la igualdad de género sigue siendo un reto fundamental para los Estados miembros y puede justificar injerencias en ciertos derechos y libertades convencionales. Ahora bien, un Estado parte no puede invocar la igualdad de género para prohibir una práctica que es defendida por las propias mujeres que la observan, en ejercicio de su libertad y sin estar sometidas a coacción, como ocurre en el caso de la demandante en S.A.S.

\section{Restricciones al ejercicio de la libertad religiosa}

Por último, se ha podido constatar prejuicios islamófobos al valorar las restricciones legítimas a la manifestación de la libertad religiosa contenidas en el segundo párrafo del artículo 9. La jurisprudencia del TEDH analiza si existe violación del derecho de libertad religiosa en dos pasos. Primero se estudia si la conducta invocada por el demandante puede considerarse una manifestación religiosa y, en un segundo momento, en el caso de que la respuesta haya sido positiva, se evalúa si el obstáculo que se denuncia como contrario a la libertad religiosa cumple los tres requisitos mencionados en el apartado

\footnotetext{
${ }^{40}$ Vid.Thomas Hammarberg, "Rulings anywhere that women must wear the burqa should be condemned-but banning such dresses here would be wrong", www.commissioner.coe.int (acceso 16-03-2018)
} 
$2^{41}$. Si los cumple, se trata de una restricción legítima a la libertad religiosa. Si no los cumple, se trata de una violación de la libertad religiosa.

Si se observan los casos que han tratado la prohibición del velo integral en Francia y en Bélgica (S.A.S, Belcacemi \& Ousar, y Dakir), el Tribunal ha concluido que las leyes de prohibiciones de carácter general, como la ley francesa y la ley belga, sólo resultarían proporcionadas si persiguen un objetivo legítimo. Y el TEDH ha afirmado que el objetivo legítimo es la protección de los derechos de los demás, que se concreta en establecer las condiciones necesarias para garantizar la convivencia (living together) entre los individuos. Para este fin, el Estado dispone de un amplio margen de apreciación en el ejercicio de su política legislativa que necesariamente se desarrolla dentro de los valores sociales vigentes en un tiempo y lugar concretos. A partir de ahí, el TEDH concluye que la cuestión de la aceptación o no del uso del velo integral en el espacio público constituye una elección de la sociedad y cuando hay en juego cuestiones de política general, "será necesario conceder una importancia particular a la función de quien lleva a cabo la decisión nacional"42. En otras palabras, Francia disponía en el caso de un amplio margen de apreciación para prohibir el velo integral por motivos de identidad nacional ${ }^{43}$.

Merece la pena destacar el voto particular discrepante de la mayoría en el caso S.A.S de las juezas Nussberger y Jäderblom que sostienen que se han sacrificado los derechos individuales por principios abstractos (living together) que no tienen correspondencia directa con los derechos y libertades garantizados en el Convenio ${ }^{44}$. Sin duda la jurisprudencia del TEDH ha señalado el deber del Estado de promover una tolerancia mutua entre los grupos opuestos y ha declarado que "la función de las autoridades (...) no consiste en eliminar la causa de las tensiones suprimiendo el pluralismo sino en vigilar que los grupos que compiten se toleren unos a otros" ${ }^{\prime 5}$. Al prohibir el velo integral, según las juezas, el legislador francés ha hecho exactamente lo contrario: lejos de garantizar la tolerancia entre una muy amplia mayoría y una pequeña minoría, ha prohibido lo que aparece como un factor de tensiones. No ha quedado demostrado que las prohibiciones hayan sido medidas necesarias y proporcionadas para la salvaguarda de la seguridad pública. Al contrario, esta legislación prohibitiva ha puesto de manifiesto hasta dónde puede el derecho doblegarse a la cultura del miedo y la importancia de las presunciones, especulaciones y estereotipos de apariencia jurídica, muy peligrosos para una democracia saludable y plural (Relaño, 2015: 33). Es decir, han eliminado de la esfera pública un tipo de manifestación de identidad cultural o religiosa que es precisamente una de las posibles manifestaciones del islam. Del razonamiento del TEDH parece desprenderse, en palabras de Thomas Hammarberg, ex Comisario de Derechos Humanos del Consejo de Europa que "en varios países europeos, el debate público se haya centrado casi exclusivamente en el carácter musulmán de la vestimenta, dando la impresión de que se tiene puesta la mira en una religión específica. Se han

\footnotetext{
${ }^{41}$ Artículo 9.2. La libertad de manifestar su religión o sus convicciones no puede ser objeto de más restricciones que las que, previstas por la ley, constituyan medidas necesarias, en una sociedad democrática, para la seguridad pública, la protección del orden, de la salud o de la moral públicas, o la protección de los derechos o las libertades de los demás.

${ }^{42}$ STEDH, S.A.S. c. Francia, par. 154.

${ }^{43}$ Ibid. par. 155.

${ }^{44} \mathrm{Ibid}$. voto particular, par. 5.

45 Ibid. voto particular, par. 14.
}

REIM № 24 (junio 2018)

ISSN: $1887-4460$ 
utilizado algunos argumentos claramente islamofóbicos, y eso no ha ayudado a tender puentes ni a favorecer el diálogo".

\section{Conclusión}

Los esfuerzos internacionales de los organismos europeos en la lucha contra la islamofobia han puesto en evidencia, precisamente, el fracaso de las medidas adoptadas desde hace décadas por los mismos organismos que reiteran, hoy en día, su compromiso para erradicar un fenómeno que va en aumento. La causa del fracaso reside tanto en el carácter no obligatorio de la mayoría de las recomendaciones, como en la ausencia de una adecuada supervisión e implantación. Las respuestas jurídicas y políticas existen, pero no son efectivas puesto que los estereotipos que inducen a una islamofobia larvada, incluso en instancias judiciales, se han extendido en la sociedad civil europea. No cabe duda que se ha ahondado en las conciencias de todos los actores y se ha crecido en la sensibilización de actores públicos como privados. Sin embargo, puede también apreciarse políticas públicas, implementadas por partidos conservadores, que han ignorado las políticas de integración y la existencia de la diferencia en nuestras sociedades. Como se ha señalado, el Consejo de Europa y la ECRI han insistido a los gobiernos a tomar medidas resolutivas y efectivas para erradicar los perjuicios negativos alimentados por el miedo al "otro" (musulmán). Estas medidas también conciernen a los operadores jurídicos que, lamentablemente, no escapan a la tendencia al estereotipo y a la fobia al islam.

Cuando el presidente del Tribunal de Luxemburgo, Lenaerts, leyó en voz alta la sentencia del caso Achbita en su lengua materna leyó "pañuelo islamista", en lugar de "pañuelo islámico". Si bien en holandés las palabras "islamista" e "islámico" se diferencian en una sola letra (Islamitisch/Islamistisch), cualquiera que tenga cierta familiaridad con los debates sobre el islam y el islamismo conoce la gran diferencia entre ambos términos. Ante este tipo de islamofobia casi "inconsciente", se hace necesario reiterar el cumplimiento de las obligaciones internacionales en todo lo relativo al respeto de la diversidad religiosa y a la lucha contra la islamofobia. Basta con volver una y otra vez a los principios básicos y vinculantes de la Constitución europea y a los pilares sobre los que se ha levantado la Unión Europea: la libertad, igualdad y el respeto por la dignidad del ser humano.

\section{Bibliografía}

AGENCIA DE LOS DERECHOS FUNDAMENTALES DE LA UNIÓN EUROPEA (FRA) (2017): Minorities and Discrimination Survey (EU-MIDIS II): Muslims - Selected findings, disponible en http://fra.europa.eu/en/publication/2017/eumidis-ii-muslims-selected-findings [consulta: 21 de febrero de 2018].

AMNISTÍA INTERNACIONAL (2012): Elección y prejuicio. Discriminación de personas musulmanas en Europa, disponible en https://www.amnesty.org/es/press-releases/2012/04/discriminacionpersonas-musulmanas-manifestar-fe/ [consulta: 20 de junio de 2018].

BAYRAKLI, Enes \& HAFEZ, Farid (2016): European Islamophobia Report 2016, Istanbul, SETA. O'NEILL, Brenda; GIDENGIL, Elisabeth \& et al. (2014): “Freedom of religion, women's agency and banning the face veil: the role of feminist beliefs in zapping women's opinión", Ethnic and Racial Studies, vol. 38, no 11, pp. 1-16.

BREMS, Eva (2017): "Analysis: European Court of Justice Allows Bans on Religious Dress in the Workplace", disponible en https://iacl-aidc-blog.org/2017/03/25/analysis-european-court-ofjustice-allows-bans-on-religious-dress-in-the-workplace/ [consulta: 16 de marzo de 2018]. 
BREMS, Eva (2013): "Face veil Bans in the European Court of Human Rights: The importance of empirical findings", Journal of Law and Policy, vol. 22, pp. 517-551.

BREMS, Eva (ed.) (2014): The Experience of Face Veil Wearers in Europe and the Law, Cambridge, Cambridge University Press.

BRAVO LOPEZ, Fernando (2011): "Towards a definition of Islamophobia: approximations of the early twentieth century", Ethnic and Racial Studies, vol. 34, no 4, pp. 556-573. https://doi.org/10.1080/01419870.2010.528440

BECTOVIC, Safet (2011): "Studying Muslims and constructing Islamic identity", Ethnic and Racial Studies, vol. 34, no 7, pp. 1.120-1.133. DOI: 10.1080/01419870.2010.528782

COMISION EUROPEA CONTRA EL RACISMO Y LA INTOLERANCIA (ECRI) (2000), Recomendación General no 5 Lucha contra la intolerancia y la discriminacion contra los musulmanes, adoptada el 16 de marzo de 2000, CRI(2000)21, Estrasburgo.

COMISION EUROPEA CONTRA EL RACISMO Y LA INTOLERANCIA (ECRI) (2003), Recomendación de política general $n$ o 7 sobre la legislación nacional para luchar contra el racismo y la discriminación racial, adoptada el 13 de diciembre de 2002, CRI(2003)8, Estrasburgo.

COMISION EUROPEA CONTRA EL RACISMO Y LA INTOLERANCIA (ECRI) (2016), Recomendación General no 15 relativa a la lucha contra el discurso de odio, adoptada el 8 de diciembre de 2015, CRI (2016)15, Estrasburgo.

CONSEJO DE EUROPA, OSCE/OIDDH, UNESCO (2012): Directrices para educadores sobre la manera de combatir la intolerancia y la discriminación contra los musulmanes: Afrontar la islamofobia mediante la educación, Varsovia, OSCE.

EVANS, Carolyn (2000): Freedom of Religion under the ECHR, Oxford, Oxford University Press.

EUROPEAN NETWORK AGAINST RACISM (ENAR) (2016): Forgotten Women: the impact of Islamophobia on Muslim women, Brussels, ENAR.

HAFEZ, Farid (2014): "Shifting borders: Islamophobia as common ground for building panEuropean right-wing unity", Patterns of Prejudice, vol. 48, no 5, pp. 479-499. https://doi.org/10.1080/0031322X.2014.965877

HALLIDAY, Fred (1999): “'Islamophobia' reconsidered”, Ethnic and Racial Studies, vol. 22, no 5, pp. 892-902. https://doi.org/10.1080/014198799329305

HOWARD, Erica (2012): "Banning Islamic veils. Is gender equality a valid argument?", International Journal of Discrimination and the Law, vol. 12, no 3, pp. 147-165.

https://doi.org/ 10.1177/1358229112464450

HUMAN RIGHTS WATCH (2010): "Belgium: Muslim Veil Ban Would Violate Rights. Parliament Should Reject Bill for Nationwide Restrictions", 21 de abril de 2010, disponible en www.hrw.org [consulta: 16 de marzo de 2018].

HUMAN RIGHTS LEAGUE (Ligue des droits de l'homme), "Interdiction du port du voile intégral: une mauvaise solution à un vrai problème", 28 de abril de 2010, http://liguedh.be [consulta: 16-032018]

KEDIKLI, Umut y Mehmet AKCA (2018): "Rising Islamophobic Discourses in Europe and Fight Against Islamophobia on the Basis of International Organizations", Mediterranean Journal of Social Sciences, vol. 9, no 1, pp. 14-17. https://doi.org/10.2478/mjss-2018-0001

MARRANCI, Gabriele (2005): "Multiculturalism, Islam and the clash of civilizations theory: Rethinking Islamophobia", Culture and Religion, vol. 5, no 1, pp. 105-117.

https://doi.org/ 10.1080/0143830042000200373 
MARTÍNEZ-TORRÓN, Javier (2003): “Los límites a la libertad de religión y de creencia en el Convenio Europeo de Derechos Humanos", Revista General de Derecho Canónico y Derecho Eclesiástico del Estado, no 2.

MINISTERIO DE INTERIOR, Informe sobre la evolución de los incidentes relacionados con los delitos de odio, http://www.interior.gob.es/web/servicios-al-ciudadano/delitos-de-odio/estadisticas [consulta: 16 de marzo de 2018].

MURDOCH, John (2012): Protecting the Right of Thought, Conscience and Religion under the European Convention on Human Rights, Strasbourg Council of Europe.

OBSERVATORIO EUROPEO DEL RACISMO Y LA XENOFOBIA (EUMC) (2006): Los musulmanes en la Unión Europea: Discriminación e islamofobia, disponible en http://fra.europa.eu/en/publication/2006/highlights-eumc-report-muslims-european-uniondiscrimination-and-islamophobia [consulta: 21 de febrero de 2018].

PALOMINO, Rafael (2016): "Igualdad y no discriminación religiosa en el Derecho de la Unión Europea. A propósito de las conclusiones en los casos Achbita y Bougnaoui", Revista Latinoamericana de Derecho y Religión, vol. 2, no 2, pp. 1-34. https://doi.org/10.776/RLDR.3.33

PETKOFF, Peter (2012): "Forum Internum and Forum Externum in Canon Law and Public International Law with a Particular Reference to the Jurisprudence of the European Court of Human Rights", Religion \& Human Rights, vol. 7, no 3, pp. 183-214. https://doi.org/10.1163/18710328-12341236

PERONI, Lourdes (2014): "Religion and culture in the discourse of the European Court of Human Rights: the risks of stereotyping and naturalizing", International Journal of Law in Context, vol. 10, no 2, pp. 195-225. https://doi.org/10.1017/S1744552314000032

RANA, Junaid (2007): "The Story of Islamophobia", Souls, vol. 9, no 2, pp. 148-161. htpps://doi.org/10.1080/10999940701382607

RELAÑO PASTOR, Eugenia (en prensa): "Religious discrimination at work: Achbita \& Bougnaoui cases", en ULADZISLAU Belavusau \& Kristin HENRARD (eds.), EU Anti-Discrimination Law Beyond Gender, Oxford, Hart.

- (2015), "Las dos caras del Dr. Jekyll: las prohibiciones del velo integral a debate" en Debates jurídicos, num. 5, disponible en http://www.rightsinternationalspain.org/es/quienessomos/19/eugenia-relano [consulta: 10 de marzo de 2018]

TARAS, Raymond (2013): "Islamophobia never stands still: race, religion, and culture", Ethnic and Racial Studies, vol. 16, no 3, pp. 417-433. https://doi.org/10.1080/01419870.2013.734388 VAKULENKO, Anastasia (2012): Islamic Veiling in Legal Discourse, Abingdon, Routledge. VICKERS, Lucy (2016): "ECJ Headscarf Series 2: The Role of Choice; and the Margin of Appreciation", Strasbourg Observers, 8 de septiembre de 2016, disponible en https://strasbourgobservers.com/2016/09/08/blog-series-the-role-of-choice-and-the-margin-ofappreciation [consulta: 16 de marzo de 2018] 